\title{
Enhanced Boiling Heat Transfer on Plain and Featured Surfaces using Acoustic Actuation
}

\author{
Thomas Boziuk, Marc K. Smith, Ari Glezer \\ Georgia Institute of Technology \\ 771 Ferst Drive, Atlanta GA 30332 \\ E-mail: ari.glezer@gatech.edu
}

\begin{abstract}
Two-phase thermal management based on submerged boiling heat transfer has received considerable attention in recent years because of its potential to achieve high heat fluxes along with its relatively simple hardware and system-level coupling. However, this attractive heat transfer approach has been hampered by the critical heat flux (CHF) limit on the maximum heat transfer, which is caused by the dynamics of the vapor bubbles that form on the heated surface and the subsequent transition to film boiling that produces a large increase in the surface temperature. Recent work at Georgia Tech has exploited low-power ultrasonic acoustic forcing to enhance boiling heat transfer and increase the CHF limit by controlling the formation and evolution of the vapor bubbles and inhibiting the instabilities that lead to film boiling. These effects are investigated with plain and textured (surface-embedded microchannels) boiling heat transfer base surfaces. Even without acoustic actuation, the transfer of makeup fluid to the boiling sites in the presence of surface microchannels passively decreases the surface superheat and increases in the CHF by $218 \%$ compared to plain surfaces. Acoustic actuation has a profound effect on the onset and evolution of boiling by inducing interfacial forces that affect the bubbles' contact line with the surface and lead to bubble detachment. Heat transfer measurements with acoustic actuation demonstrate a significant increase in the CHF as the acoustic field impedes the formation of large vapor columns and inhibits the instability that results in the collapse of these columns into a vapor film. The improvements in the CHF in stagnant bulk fluid exceed $66 \%$ for the plain surface (up to $183 \mathrm{~W} / \mathrm{cm}^{2}$ ), and $31 \%$ for the textured surface (up to $460 \mathrm{~W} / \mathrm{cm}^{2}$ with a $7{ }^{\circ} \mathrm{C}$ reduction in surface superheat).
\end{abstract}

KEY WORDS: boiling, heat transfer, microchannel, acoustic, critical heat flux, ultrasound

\section{NOMENCLATURE}

CHF Critical Heat Flux, W/ $\mathrm{cm}^{2}$

$\Delta T_{\mathrm{s}} \quad$ Change in Surface Superheat under Actuation, ${ }^{\circ} \mathrm{C}$

$T_{\mathrm{s}} \quad$ Surface Temperature, ${ }^{\circ} \mathrm{C}$

$T_{\text {sat }} \quad$ Saturation Temperature of Liquid, ${ }^{\circ} \mathrm{C}$

$q, \quad$ Heat Flux, W/ $\mathrm{cm}^{2}$

$t \quad$ Time, seconds

(C) 2016. This manuscript version is made available under the Elsevier user license http://www.elsevier.com/open-access/userlicense/1.0/ 


\section{Greek symbols}

$\theta \quad$ Azimuthal Angle of Acoustic Actuation relative to Heater Plane, degrees

$\phi \quad$ Incident Angle of Acoustic Actuator relative to Heater Plane, degrees

\section{INTRODUCTION}

The increased power of integrated circuits has led to increased heat dissipation requiring a more effective means of heat transfer to avoid prohibitively high circuit temperatures. Cooling requirements for advanced integrated circuits have been projected to exceed $1,000 \mathrm{~W} / \mathrm{cm}^{2}$ [1]. To accommodate such large heat fluxes, thermal management technologies for electronic hardware have begun to rely on single- and two-phase liquid cooling [2][3]. In single-phase cooling, liquids provide higher thermal conductivity than gases, which enhances natural and forced convection, but two-phase cooling significantly enhances heat transfer owing to the heat absorption of liquid-to-gas phase change. Furthermore, the vapor bubbles that form on and subsequently detach and advect from the hot surface into the bulk liquid induce fluid motions that promote enhanced convection. However, boiling heat transfer is limited by a transition from nucleate to film boiling at a critical level of heat flux beyond which an insulating vapor film develops over the heated surface. This results in a dramatic increase in the surface temperature that may severely damage the cooled electronic hardware.

A number of earlier investigations focused on enhancement of boiling heat transfer by facilitating the detachment of the vapor bubbles from the heated surface with the objectives of improving the heat transfer from the surface at a given surface temperature and, in some cases, increasing the critical heat flux (CHF) limit. These effects have been demonstrated by affecting the vapor bubbles' evolution either passively or actively. Passive effects can be accomplished through geometrical modification of the heated surface which in turn can affect bubble nucleation and growth. Active forcing can be applied directly by using surface forces engendered by a cross flow, or indirectly, by using body forces effected by acoustic actuation.

The effects of surface geometry on the formation, evolution, and detachment of vapor bubbles have been reported in a number of earlier investigations. Relatively simple surface designs based on traditional evaporator/condenser fin geometries were driven by the desire to reduce the characteristic dimensions of the heat transfer surfaces. McGillis and Carey [4] performed a parametric study of a rectangular fin array having characteristic pitch, height, and fin thickness of 1.5, 5.0, and $2.7 \mathrm{~mm}$, respectively and reported an increase in CHF of up to $150 \%$. These authors also concluded that the fin thickness was not an important parameter, noting that the fin length should only be about 1.4 times larger than the fin spacing. In a review article of the effects of surface geometry on boiling heat transfer, Bergles [5] noted that while surface geometry can have a profound effect on boiling heat transfer processes, understanding the underlying physical mechanisms is necessary for the development of new generations of surface-induced boiling enhancements. More recently, more complex surface designs have included nano-scale deposits of copper tubes that increase the wettability of the copper surface as well as the nucleation site density [6]. In a hybrid 
micro-nano-structure, Launay et al. [7] used carbon nanotubes grown onto a grooved silicon heat sink to produce improvements at multiple scales. They found that nanotubes improved performance primarily at low heat fluxes $\left(<15 \mathrm{~W} / \mathrm{cm}^{2}\right)$, while microscale grooves provided the majority of improvement at higher heat fluxes (up to $130 \mathrm{~W} / \mathrm{cm}^{2}$ ). Chien and Webb [8] worked with subsurface $0.35 \mathrm{~mm}$ wide mm-scale channels, and noted that an ample supply of liquid to the evaporating surface was crucial for attaining the highest heat transfer coefficients $\left(2 \mathrm{~W} / \mathrm{K} / \mathrm{cm}^{2}\right)$ that were afforded by the surface geometry. Recent investigations of heat transfer in laminated-screen surfaces $[9,10]$ has shown that layers of copper mesh bonded to the heat transfer surface can increase CHF and the stability of the wall temperature along the boiling curve.

The drag force induced on vapor bubbles by a cross flow of a coolant over the heated surface has been exploited for forced detachment. However, such heat transfer improvement comes at a cost in the power, weight, and size of the cooling hardware [11]. Some of these limitations can be overcome by using cross flow that is induced by submerged synthetic jets that require no external fluid source $[12,13]$. The use of body forces effected by an ultrasonic acoustic field for indirect vapor bubble removal has been investigated by a number of authors including Isakoff [14], Wong and Chon [15], Park and Bergles [16], and Kim et al. [17]. These works demonstrated that ultrasound can lead to the creation of small cavitation bubbles that enhance mixing in the bulk liquid and thereby increase natural convection heat transfer near the onset of boiling with limited effects on nucleate boiling and a slight increase in the critical heat flux. The cavitation bubbles are apparently overwhelmed by the appearance of the natural vapor bubbles that form during nucleate boiling. Sitter et al. $[18,19]$ examined boiling on a wire in the presence of an acoustic field in terrestrial and microgravity experiments and found that the acoustic actuation led to an increase in the heat transfer coefficient on the wire by directly coupling with the natural oscillations of vapor bubbles through the action of the primary Bjerknes force. Hao et al. [20] investigated the effect of the primary and secondary Bjerknes forces on a vapor bubble near a planar rigid wall using a numerical simulation and proposed that these acoustic effects could be used to improve boiling heat transfer from a horizontal surface. Douglas et al. [21] reported the effect of acoustic actuation on the contact line of pinned air and vapor bubbles, as well as the critical heat flux of submerged boiling over a plain copper surface. Li et al. [22] studied the effect of acoustic fields (21 and $45 \mathrm{kHz})$ on boiling over the surface of smooth and textured (azimuthal and spiral fins) cylindrical tubes and showed that the presence of the textured surfaces increased the acoustic enhancement in heat transfer by up to $42 \%$ increase compared to $16 \%$ over smooth tubes. In a related investigation of highly subcooled $\left(80^{\circ} \mathrm{K}\right)$ submerged boiling on wire heaters (characteristic diameters $\left.50-250 \mu \mathrm{m}\right)$ mounted in tension in a $40 \mathrm{kHz}$ acoustic field, Hetrosini et al. [23] found that the acoustic field induced wire vibrations that decrease with increasing wire diameter leading to heat transfer enhancement with a maximum reduction of $40^{\circ} \mathrm{K}$ in the temperature of a $200 \mu \mathrm{m}$ wire.

The present paper discusses heat transfer enhancement and the extension of the critical heat flux limit of boiling on submerged plain and textured heated surfaces using acoustic actuation. The actuation has a profound effect on the creation and 
evolution of the vapor bubbles by inducing interfacial forces that affect the bubbles' contact line with the surface and accelerates their detachment. The experimental set up and techniques are described in Section 2. The heat transfer characteristics of microchannels embedded in the heater's surface are compared with the characteristics of a plain heater in Section 3. The effects of ultrasonic actuation on global heat transfer from a plain-surface heater and on individual nucleation sites are discussed in Sections 4 and 5, respectively. Enhancement of heat transfer from a surface textured with microchannels is described in Section 6. Finally, concluding remarks are presented in Section 7.

\section{EXPERIMENTAL APPARATUS AND PROCEDURE}

The boiling process on a submerged calibrated heated surface is investigated in a dedicated water test cell shown schematically in Figure 1. The heater comprises a cartridge heating element that is inserted within a copper cylinder and is instrumented with two sets of three thermocouple sensors each along a vertical line $5.5 \mathrm{~mm}$ off the axis that are $120^{\circ}$ apart azimuthally, for measurements of time-dependent heat flux and estimates of the heater's surface temperature (within the frequency response of the sensors). The top segment of the cylindrical heater assembly $(1.6 \mathrm{~cm}$ diameter $)$ that is the boiling surface in contact with the liquid pool is flush-mounted within and sealed to a cylindrical ceramic module that forms the center segment of the bottom surface of the test cell. This heater segment is designed to be interchangeable for testing the effects of plain (baseline) and textured boiling surfaces on the nucleation and evolution of vapor bubbles. One of the plain removable surface heater segments is instrumented with three surface-soldered thermocouple sensors for direct of superheat temperatures at the boiling surface. The three miniature ( $250 \mu \mathrm{m}$ diameter) stainless steel sheathed thermocouple sensors are embedded such that each sensor bead is flush with the boiling surface, and are equally-spaced along a $12 \mathrm{~mm}$ diameter circle. Textured surfaces
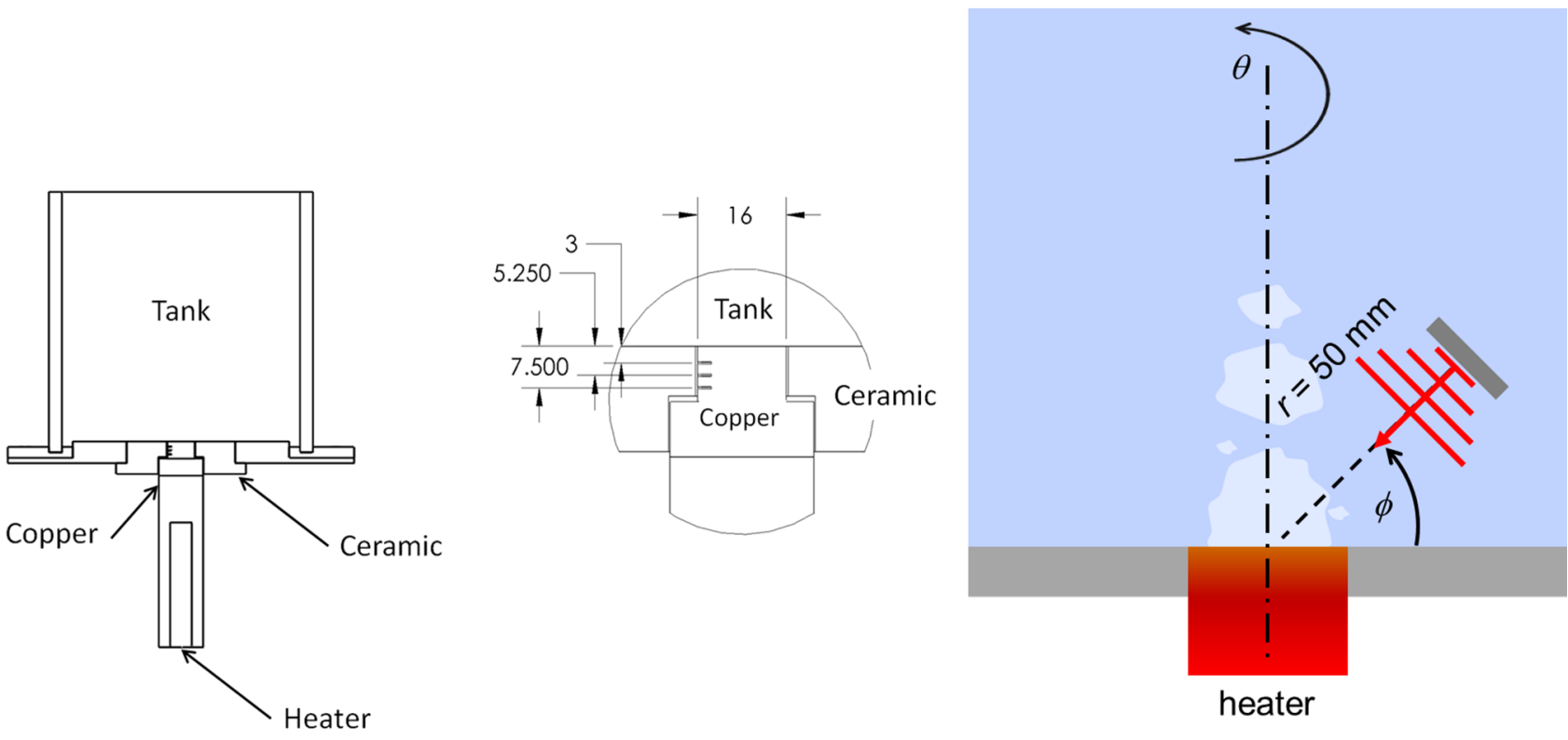

Figure 1. The submerged boiling test cell with a calibrated heater (left), a schematic of the calibrated heater with data in $\mathrm{mm}$ (center), and the position of the acoustic transducer relative to the heated surface (right). 
are formed by a square grid of intersecting open-surface machined microchannels with widths varying from 200 to $1000 \mu \mathrm{m}$, and depths of up to $1.8 \mathrm{~mm}$ (see Figure 2 inset). These microchannels enable liquid flow into nucleation sites at the intersections for enhanced heat transfer.

As noted above, the surface temperature of and the heat flux through the heater is normally estimated using the two sets of the three embedded thermocouple sensors along the heater's cylinder using a linear extrapolation based on 1-D heat transfer. For this heater assembly, the maximum uncertainties of the surface temperature and heat flux are estimated to be $\pm 0.4{ }^{\circ} \mathrm{C}$ and $\pm 7 \%$, respectively. The estimated temperature was compared directly with the temperature that was measured by the surface soldered thermocouples at a baseline boiling condition of $100 \mathrm{~W} / \mathrm{cm}^{2}$. This comparison showed that the extrapolated surface temperature (nominally $115^{\circ} \mathrm{C}$ ) was $1.7^{\circ} \mathrm{C}$ above the measured surface temperature (averaged over the three surface-soldered thermocouple sensors) or about $0.44 \%$. In addition, a numerical simulation of axisymmetric heat transfer through the entire heater was used to compare a simulated extrapolated surface temperature (using the actual thermocouple locations and a onedimensional heat flux assumption) to the simulated actual surface temperature taking into account three-dimensional heat transfer effects. This simulation showed that at a surface heat flux of $100 \mathrm{~W} / \mathrm{cm}^{2}$ the extrapolated surface temperature was about $1.8^{\circ} \mathrm{C}$ higher than the measured surface temperature, while the (one-dimensional) heat flow was underestimated by about $3 \%$ [21]. Therefore, it may be concluded that the one-dimensional heat flux is an acceptable approximation.

A secondary immersion heater is used to control and regulate the bulk temperature in the tank. In the present experiments, the nominal bulk temperature is $93{ }^{\circ} \mathrm{C}$, yielding $7{ }^{\circ} \mathrm{C}$ subcooling at atmospheric pressure. The procedures for polishing, cleaning, and preparation of the heater surfaces prior to boiling experiments is described in Douglas et al. [21].

Acoustic actuation is provided by a $20 \mathrm{~mm}$ diameter, $1.7 \mathrm{MHz}$ ultrasonic piezoelectric transducer that is driven by an external amplifier. The transducer is mounted in the tank above the heated surface using an articulated support such that the acoustic beam can be directed at the heated surface over a range of angles as shown schematically in Figure 1. The acoustic pressure along and normal to the centerline of the transducer was measured using a calibrated submerged hydrophone (CTSValpey Pinducer type VP-1063) at several driving voltages yielding maximum peak-to-peak pressure fluctuations of $6.82 \cdot \times 10^{3}$ $\mathrm{kPa}$ at a distance of $50 \mathrm{~mm}(6 \%$ uncertainty). At this axial location the peak-to-peak pressure decreases by $25 \%$ and $63 \%$ at 2 and $4 \mathrm{~mm}$ away from the centerline respectively. The wavelength for a $1.7 \mathrm{MHz}$ acoustic field in water is only $\sim 0.9 \mathrm{~mm}$, which is smaller than the majority of the vapor bubbles, and therefore causes a non-uniform pressure field around each bubble. Additionally, this small wavelength allows ultrasonic actuation to remain effective on structured surfaces, whose small-scale features reduce the effect of larger-wavelength acoustic fields. A $1.7 \mathrm{MHz}$ natural frequency corresponds to a bubble diameter of $\sim 2 \mu \mathrm{m}$, so phenomena that depend on bubble resonance (for example, the Bjerknes force) are almost nonexistent [24]. Additionally, the capillary wavelength is so small $(5 \mu \mathrm{m})$ that surface waves, though not resolved by the CMOS camera (see 
below), are likely negligibly small in amplitude due to immediate damping at the interface [25]. The $1.7 \mathrm{MHz}$ acoustic field provides a useful effect because of its focused, high-intensity beam. The radiation pressure of the acoustic beam due to the mismatch in acoustic impedance between the vapor and liquid phase of water results in a force on bubble interfaces, leading to motion of vapor bubbles away from the actuator. This force is referred to by Torr [26] as radiation pressure that can lead to deformation of liquid/vapor interfaces [27]. At these acoustic frequencies, the effects of the radiation pressure are much larger than the effects of the Bjerknes force [21]. The Bjerknes force is associated with bubble resonance at the acoustic actuation frequency. In the present experiments the scale of the bubbles for which the Bjerknes force is significant is $\mathrm{O}[1 \mu \mathrm{m}]$, and considerably smaller than the scale of the vapor bubbles that form on the heater. This characteristic of the acoustic actuation distinguishes the present investigations from prior works that utilized significantly lower acoustic frequencies.

The dynamics of the boiling process over the surface is captured using high-magnification, high-speed video imaging. The present high-speed CMOS video camera can resolve 800 x 600 pixels up to $10,000 \mathrm{fps}$.

\section{THE EFFECTS OF SURFACE MICROCHANNELS ON SUBMERGED BOILING}

Boiling curves were generated for several plain and textured copper heater segments using a quasi-steady incremental increase in heat flux until the critical heat flux was reached (Figure 2). This procedure is decribed in detail in Douglas et al. [21]. The surface of the plain, smooth segment (the baseline heater) is polished to provide contact angles of nearly $90^{\circ}$ for isolated vapor bubbles, and the heat transfer curve (Figure 2, black curve) shows that a CHF of $110 \mathrm{~W} / \mathrm{cm}^{2}$ is reached at a surface superheat of $17{ }^{\circ} \mathrm{C}$, when nucleate boiling completely transitions to film boiling. Since entrainment of subcooled

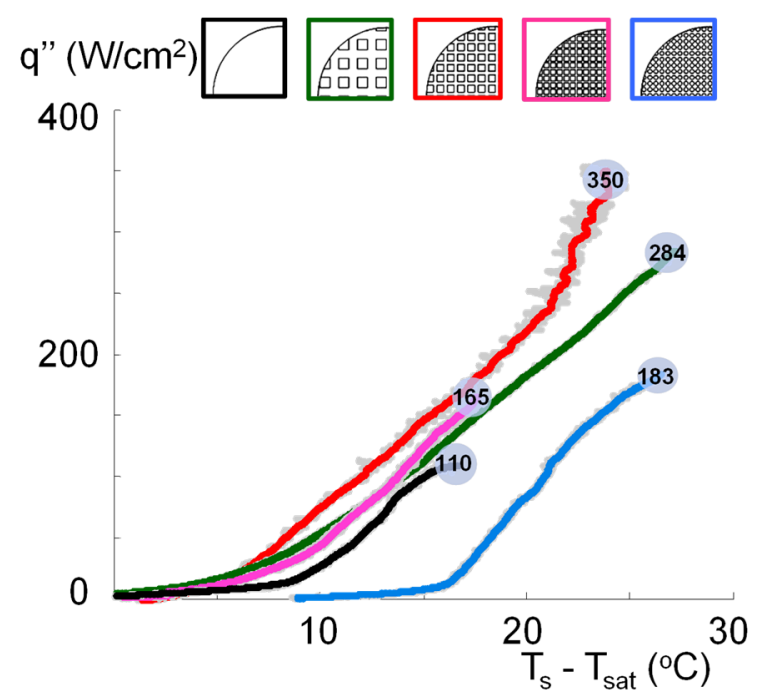

Figure 2. Boiling curves for several textured heat transfer surfaces shown at the top (the wetted areas are also noted): plain $200 \mathrm{~mm}^{2}$; microchannels: $200 \mu \mathrm{m}, 570 \mathrm{~mm}^{2}, 400$ $\mu \mathrm{m}, 600 \mathrm{~mm}^{2}$, and $1000 \mu \mathrm{m}, 520 \mathrm{~mm}^{2}$; and dimpled 600 $\mu \mathrm{m}, 825 \mathrm{~mm}^{2}$. ambient liquid is induced by the vaporization process, CHF is reached when the transport of the subcooled fluid to the vapor bubbles and nucleation sites is either insufficient or disrupted by the rapid formation of the bubbles. In the present investigations, textured surfaces are designed to passively transport subcooled liquid to the nucleation sites in order to sustain the evaporation and prevent local dryouts that can spread and transition to film boiling and lead to CHF. This is accomplished using a square grid of intersecting, open-surface microchannels that provide a regular array of nucleation sites at the intersections while transporting subcooled liquid to these sites by exploiting the flow induced by rising vapor bubbles. 
Furthermore, spreading of local dryout spots is reduced by segregating vapor production at the surface to distinct locations within the microchannels. The present experiments use three microchannel grids of different width/pitch/depth with reasonably close wetted areas: $200 \mu \mathrm{m} / 0.8 \mathrm{~mm} / 0.5 \mathrm{~mm}\left(570 \mathrm{~mm}^{2}\right), 400 \mu \mathrm{m} / 1.2 \mathrm{~mm} / 1.0 \mathrm{~mm}\left(600 \mathrm{~mm}^{2}\right)$, and $1000 \mu \mathrm{m} / 2.2 \mathrm{~mm} / 1.8 \mathrm{~mm}$ $\left(520 \mathrm{~mm}^{2}\right)$. A grid of hemispherical dimples (nominally $1 \mathrm{~mm}$ deep) to anchor vapor bubbles was also included for comparison. The heat flux is computed using the projected area $\left(2 \mathrm{~cm}^{2}\right.$ for all surfaces $)$ and the surface temperature is extrapolated to the bottom of the channels, which corresponds to the hottest wetted surface. For each geometry, a heat transfer curve up to the critical heat flux is generated as shown in Figure 2. These data show that the surface with $400 \mu \mathrm{m}$ channels is most effective for reducing the surface superheat and yielding the highest CHF (up to $350 \mathrm{~W} / \mathrm{cm}^{2}$ ). It is noteworthy that the dimpled surface, which had nucleation sites but no organized fluid transport, has a higher surface superheat compared to the baseline (plain) surface ostensibly because vapor fills the cavities with limited replenishing of fluid resulting in an insulation of some surface fraction and therefore raising the mean surface superheat for a given heat flux. The "optimal" $400 \mu \mathrm{m}$ channel represents a balance between the flow rate of subcooled liquid through the channel and its preheating which aids evaporation, which increases and decreases with increasing channel width, respectively.

\section{BOILING ENHANCEMENT ON A PLAIN BOILING SURFACE USING ULTRASONIC ACTUATION}

The effects of acoustic actuation with an incidence angle of $45^{\circ}$ on the evolution of vapor bubble clusters over a range of heat fluxes up to the critical heat flux is first investigated using a plain boiling surface. The boiling curves with and without actuation are shown in Figure 3. These data show that actuation increases the surface superheat by up to $7{ }^{\circ} \mathrm{C}$ relative to the baseline, and, perhaps more significantly, increases the CHF from 110 to $183 \mathrm{~W} / \mathrm{cm}^{2}$, an improvement of $65 \%$.

The evolution of vapor bubbles on the surface of the baseline heater was investigated using high-speed video imaging at

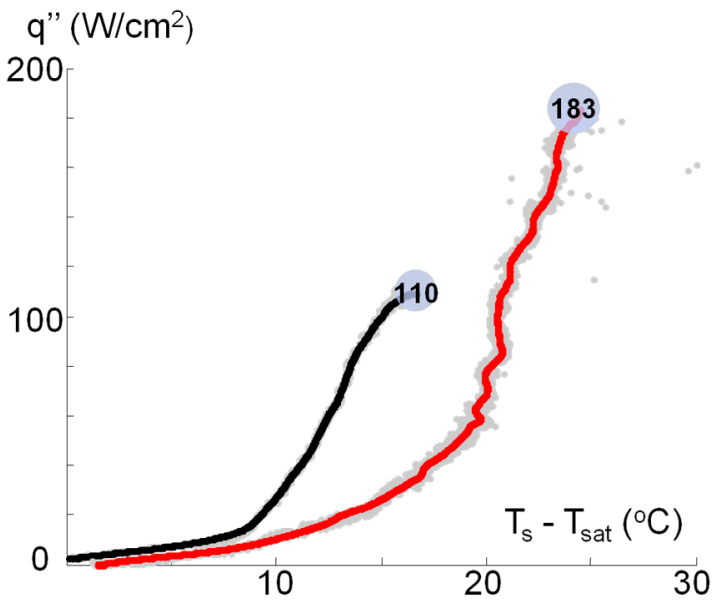

Figure 3. Boiling curves for the plain (baseline) surface in the absence and presence of acoustic actuation. Blue insets denote the levels of the critical heat flux.
10,000 fps. Figures $4 \mathrm{a}-\mathrm{e}$ and $4 \mathrm{f}-\mathrm{j}$ show images of the response of vapor formation in the absence and presence of ultrasonic actuation, respectively, at five levels of power dissipation (between 25 and $\left.110 \mathrm{~W} / \mathrm{cm}^{2}\right)$. At low power levels $(25-$ $75 \mathrm{~W} / \mathrm{cm}^{2}$ ), the actuation clearly results in lower vapor volume on the surface of the heater indicating an increase in the forced detachment of small-scale bubbles that form at various nucleation sites. Furthermore, it is clear that evaporation is diminished on the plain surface and is restricted to fewer ("preferred") nucleation sites such as those along the edge of the 

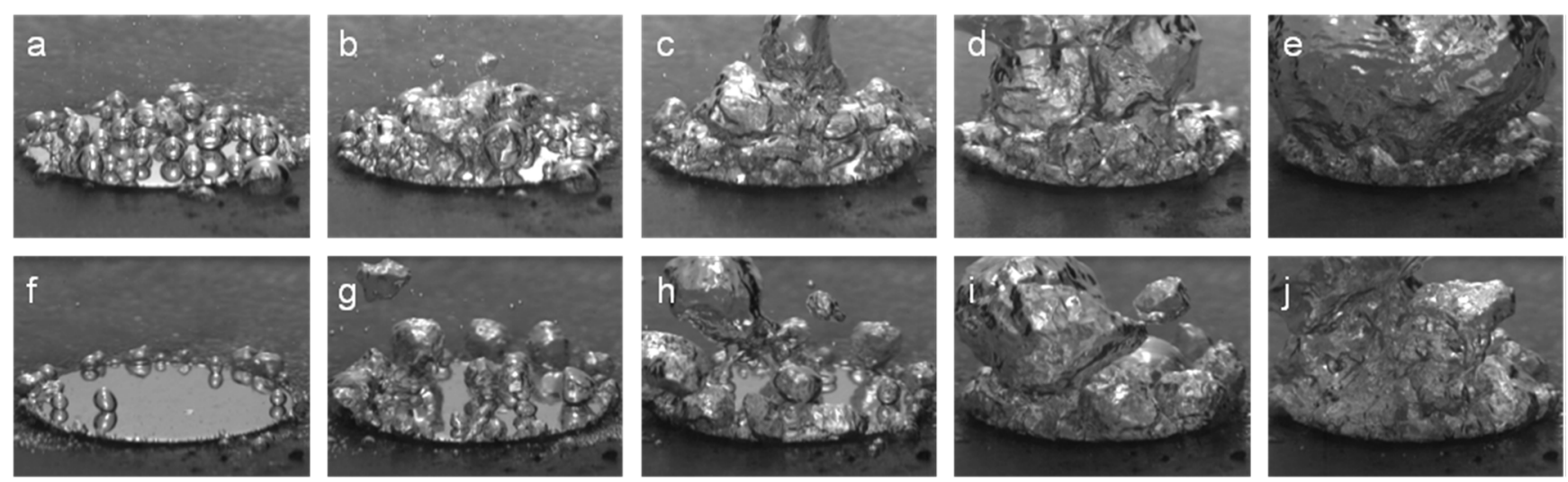

Figure 4. Images from high-speed videos of vapor formation along the heat transfer curves of the baseline surface (Figure 3 ) in the absence (top row, a-e) and presence of acoustic actuation (bottom row, $f-j$ ) at 25, 50, 75, 100, and 110 W/cm respectively.

heater (Figure 4f). The differences in vapor mass above the heater surface at higher power levels do not appear to be very significant in Figure 4, but the larger-scale images in Figure 5 clearly indicate that ultrasonic actuation significantly alters the structure of the vapor column above the heater surface. Figures 5a-f and 5g-1 show sequences of images captured at moderate heat flux levels $\left(100 \mathrm{~W} / \mathrm{cm}^{2}\right)$ in the absence and presence of actuation, respectively. In the absence of actuation (Figures 5a-f), a large vapor mass forms over the baseline heated surface and the images show a regular, almost time-periodic formation and liftoff of the vapor mass, which also causes entrainment of subcooled liquid underneath the vapor through the action of buoyancy force (see also Figures $4 \mathrm{e}$ and $\mathrm{j}$ ). However, in the presence of actuation (Figure $5 \mathrm{~g}-1$ ) the volumes of the vapor mass and of the bubbles that lift off are considerably smaller, and the smaller-scale vapor bubbles that lift off are advected away at a higher rate. The smaller vapor volumes in these images also seems to be the result of enhanced condensation of the vapor mass at the vapor-liquid interface in the

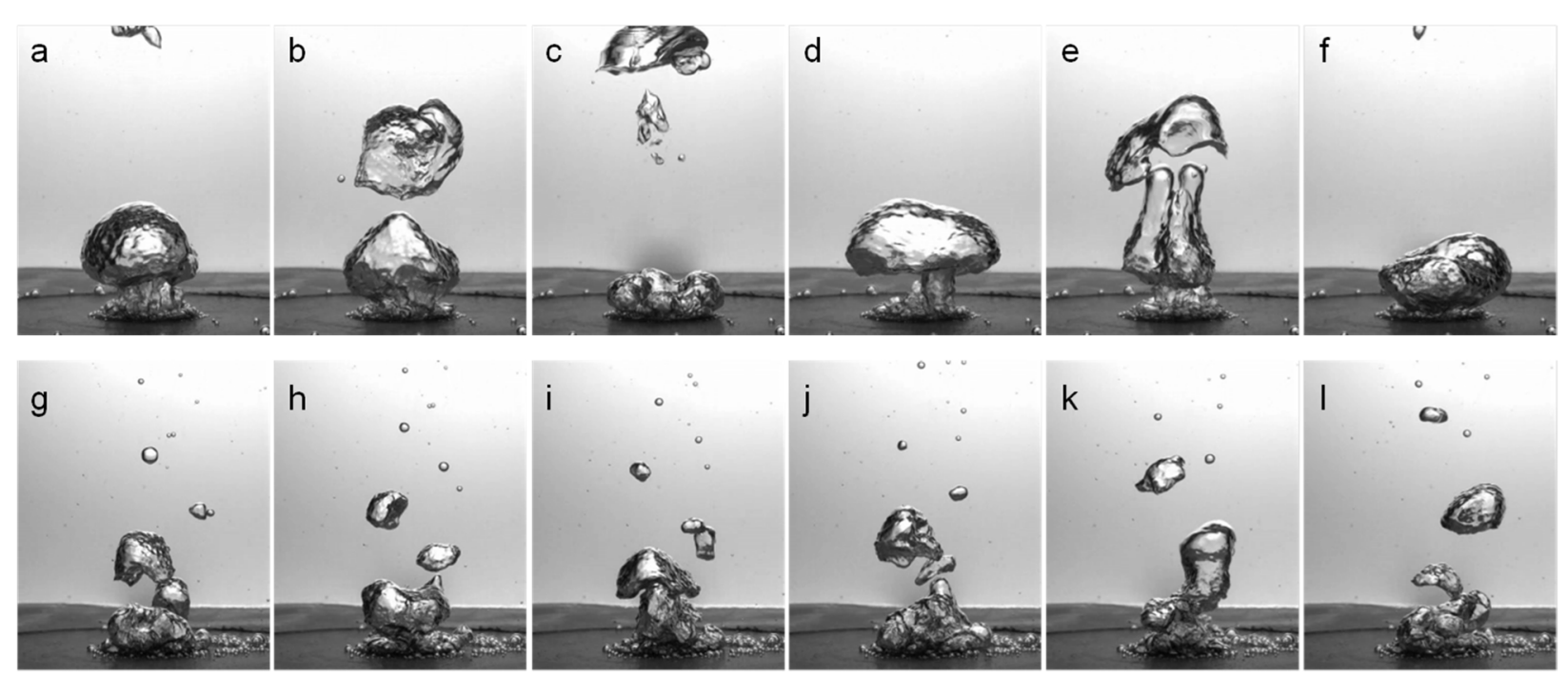

Figure 5. Vapor column formation over the baseline surface at $100 \mathrm{~W} / \mathrm{cm}^{2}$ in the absence (a-f) and presence (g-l) of acoustic actuation. The total elapsed time in the absence and presence of actuation is selected to be 240 and $100 \mathrm{msec}$, respectively in order to show details of the effects of the acoustic actuation. 

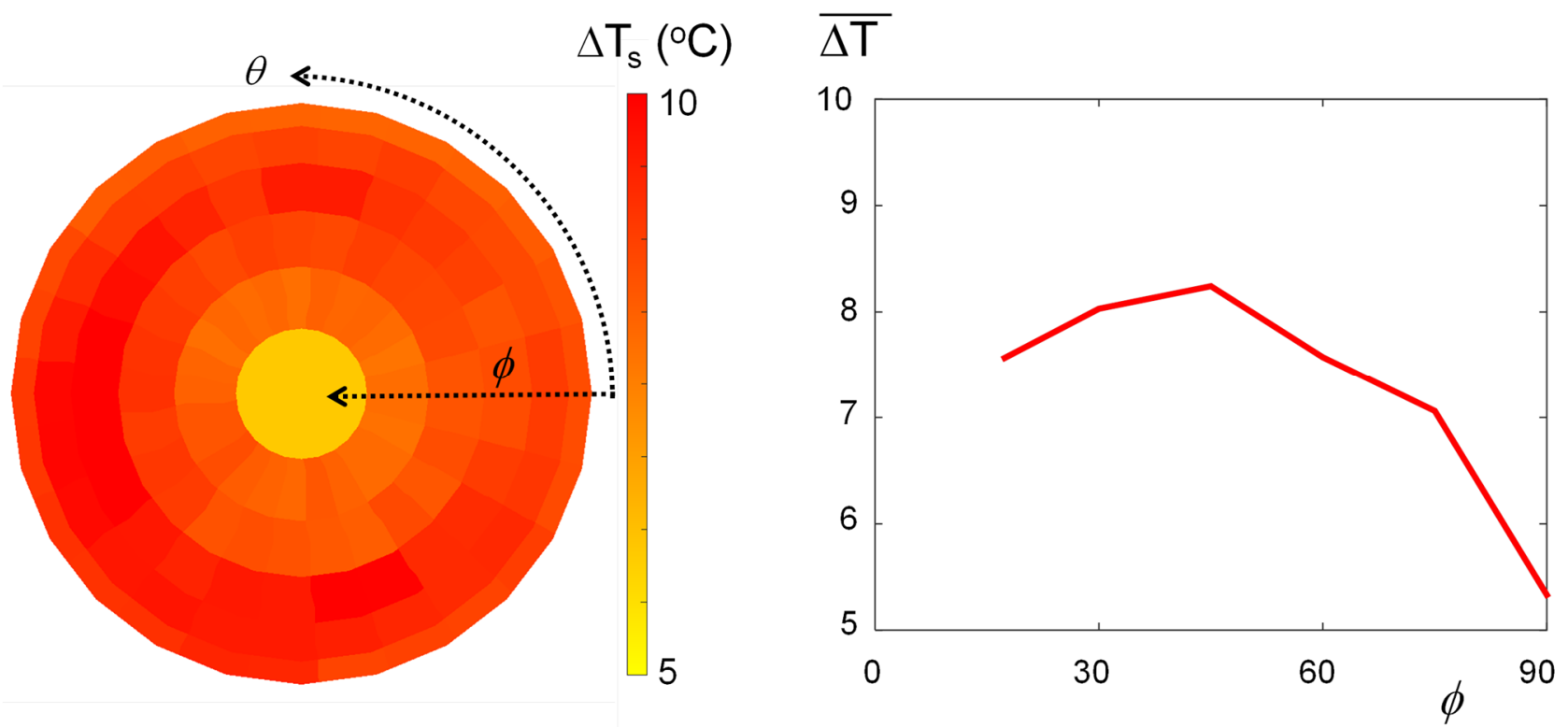

Figure 6. Contour map of surface superheat $\Delta T_{\mathrm{s}}$ for 25 equally spaced azimuthal actuator angles, and 6 incident actuator angles $\left(17^{\circ}, 30^{\circ}, 45^{\circ}, 60^{\circ}, 75^{\circ}, 90^{\circ}\right)$, with acoustic actuation. Azimuthally averaged results plotted to right.

vicinity of the heater and along the vapor column ostensibly owing to the motion of the interface caused by the acoustic radiation pressure.

A mechanism for the effect of ultrasonic actuation on the boiling curve can be conjectured based on the high-speed images from Figures 4 and 5. The radiation pressure of the acoustic beam contributes to the removal of small $[\mathrm{O}(10 \mu \mathrm{m})]$ vapor bubbles from their nucleation sites before they can grow significantly, and the increases in local pressure fluctuations and time-averaged local pressure induced by the beam suppresses evaporation at multiple nucleation sites. Both of these effects decrease the local rate of vapor formation and so the corresponding portion of the heat flux from the heater surface that supported evaporation is driven into the liquid phase. This causes the increase in the surface temperature seen in Figure 3. At the same time, the vapor mass above the surface is reduced in size by the removal of small-scale vapor bubbles from the heated surface and the accelerated condensation caused by the radiation pressure. Together, these effects contribute to the increase in the CHF limit.

The dependence of the actuation effectiveness on the incidence and azimuthal angles of the acoustic beam relative to the boiling surface were measured using the articulated actuator traverse ( $c f$. Section 2). In these measurements, the effectiveness of the ultrasonic actuation is assessed using the measured surface superheat $\Delta T_{\mathrm{s}}$ when the heater power is fixed at $100 \mathrm{~W} / \mathrm{cm}^{2}$ and the actuator's center is held at $50 \mathrm{~mm}$ away from the heated surface and the center of the beam is directed at the center of the heated surface ( $c f$. Figure 2). The variation of the surface superheat is depicted using a polar color raster plot of $\Delta T_{\mathrm{s}}$ for 25 equally spaced azimuthal angles $\theta$ (around the perimeter) and 6 incidence angles (varying radially) as shown in Figure 6, left. These data show that the changes in surface superheat are nearly invariant with the azimuthal position of the transducer. For transducer incidence angles 
$17^{\circ}<\phi<75^{\circ}$, the azimuthally-averaged $\Delta T_{\mathrm{s}}$ varies between 7 and $8.25^{\circ} \mathrm{C}$ (Figure 6, right). For $\phi>75^{\circ}$ there is a reduction in the effect of the actuation, and at $\phi=90^{\circ}$ the azimuthally-averaged $\Delta T_{\mathrm{s}}$ is $5.4^{\circ} \mathrm{C}$.

\section{ACTUATION EFFECTS AT DISCRETE BOILING NUCLEATION SITES}

The spatial effects of acoustic actuation on the surface superheat are explored using the plain boiling surface with the heater power held fixed at $100 \mathrm{~W} / \mathrm{cm}^{2}$. In these experiments, the surface superheat of the plain heater is measured directly using the azimuthal array of three miniature ( $250 \mu \mathrm{m}$ diameter) thermocouple sensors (cf. Section 2). The acoustic actuator is mounted at an incidence angle of $\phi=60^{\circ}$ and a distance of $50 \mathrm{~mm}$ from the surface, with its position offset such that the point of incidence of the centerline of the acoustic beam on the surface coincides with the radius of the circle defined by the three thermocouple sensors (defined as TC-1, TC-2, and TC-3). The acoustic beam was placed at six azimuthal positions around this circle, three of which (positions 1, 3, and 5 in Figure 7, top right schematic diagram) coincide with the radial locations of the thermocouple sensors and the other three azimuthal positions (positions 2, 4, and 6 - Figure 7, bottom right diagram) are equally spaced between the thermocouple sensors. In this way, the effect of the acoustic actuation at any given sensor position is measured when the beam is directed at the sensor and diametrically opposed to it. Phase-locked time traces of the temperature measured by each thermocouple sensor were sampled in the absence and presence of the actuation when the actuation was turned on during a pulse trigger. In addition, the surface boiling at each of the six positions was imaged using high-speed video (2,000 fps) along with simultaneous recording of the surface temperature.

Figure 7 shows phase-locked temperature time traces of the three thermocouple sensors before, during, and following the pulsed actuation corresponding to each of the six azimuthal positions of the acoustic beam along the circle. The top row
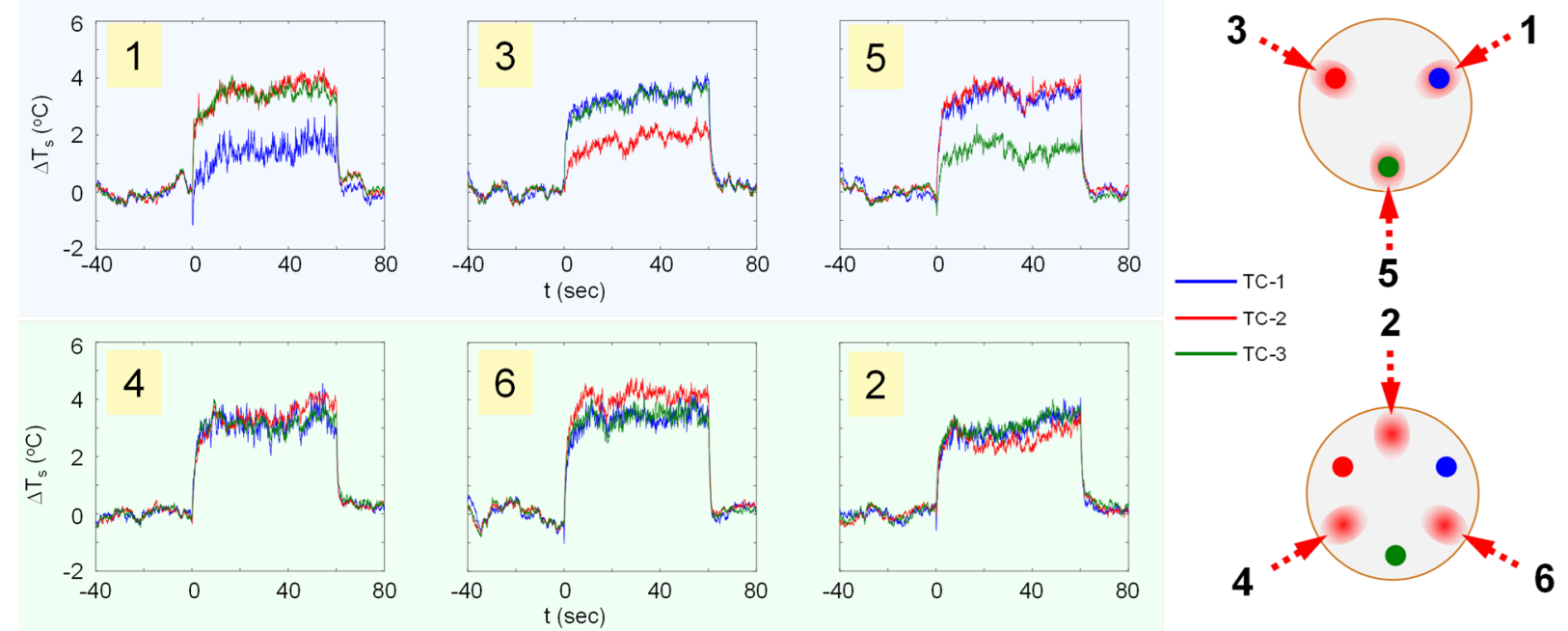

Figure 7. Time traces of surface superheat $\Delta T_{\mathrm{s}}$ measured by the three surface thermocouple sensors TC-1, 2, and 3 during onset and termination of actuation for six azimuthal position of the acoustic beam on the heater surface shown schematically by a red round spot on the right. At each set of three odd- and even-number equally-spaced azimuthal positions the acoustic beam is incident upon or between TC-1, 2, and 3, respectively. 
$\Delta \mathrm{T}_{\mathrm{s}}\left({ }^{\circ} \mathrm{C}\right)$

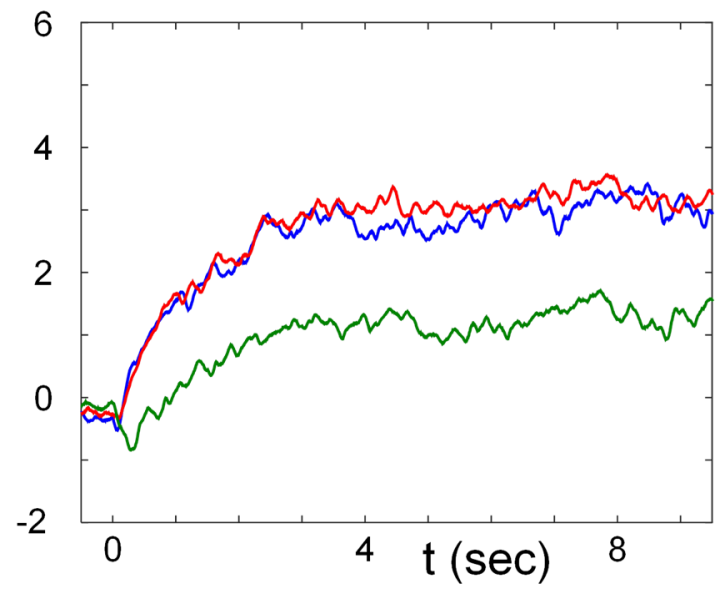

Figure 8. Time trace of surface superheat $\Delta T_{\mathrm{s}}$ measured by thermocouple sensors TC-1, 2, and 3 during the onset of actuation when the centerline of the acoustic beam is incident on TC- 3 at $\phi=60^{\circ}$. The effects on TC-1 and 2 are symmetric. (positions 1, 3, and 5) corresponds to incidence on one of the thermocouple sensors, while the bottom row (positions 4, 6, and 2) corresponds to incidence between two of the thermocouple sensors. It is clear that when the incidence of the acoustic beam is between the thermocouples (Figure 7, bottom row) the effect of the actuation on surface superheat at all locations of the thermocouple sensors is uniform, exhibiting the same nominal rise in surface superheat. Interestingly, this change in surface superheat of $3-4{ }^{\circ} \mathrm{C}$ is lower than the change of $7-8{ }^{\circ} \mathrm{C}$ that is expected from the global surface results in Figure 6. As observed in the high-speed video images in Figure 4 (for a heater surface with no surface-embedded thermocouples), vapor suppression is most noticeable on the smooth segment of the heater, with the few remaining "preferred" nucleation sites located at the edge of the heater. Surface-embedded thermocouple sensors in the heater surface also act as preferred nucleation sites where vapor formation is not completely extinguished in the presence of actuation. Hence, the additional vapor formation effectively cools the surface and decreases the local temperature rise by about $4 \mathrm{C}$.

When the centerline of the acoustic beam is incident on a thermocouple sensor (Figure 7 , top row), the change in the local surface superheat at this sensor location is significantly reduced to about 1-2 C. However, the adjacent thermocouple sensors measure the same superheat levels (3-4 C) as when the beam incidence is diametrically opposed to the sensor locations (i.e., azimuthally between adjacent sensors). This indicates that there is more vapor formation at the thermocouple sensor that is directly in the path of the beam than at the other two locations. Figure 8 shows temperature time traces of the thermocouples sampled at higher resolution. The green trace for TC-3 shows that when the incident beam strikes the thermocouple it experiences an initial almost $1 \mathrm{C}$ drop below the saturation temperature. This temperature drop is evidence of an initial, complex acoustic interaction with the vapor bubbles at the nucleation site that promotes vapor formation.

This interaction is also seen in the corresponding high-speed video images of the process. Figure 9 shows a video time series of the onset (Figure 9a-f) of acoustic actuation on the boiling surface when the beam centerline is incident on sensor TC-3 (centered in the field of view near the edge of the heater that is closest to the camera, denoted with a green dot; TC-1 is denoted in blue and TC-2 in red). There is a clear increase in vapor mass in Figure 9b relative to the baseline (Figure 9a) that is advected away before vapor suppression is evident in Figure 9f. High-speed video frames at a later time when the boiling reaches an asymptotic state (Figures 9g-1) show significant transitory evaporation at the incident thermocouple sensor. This transitory 

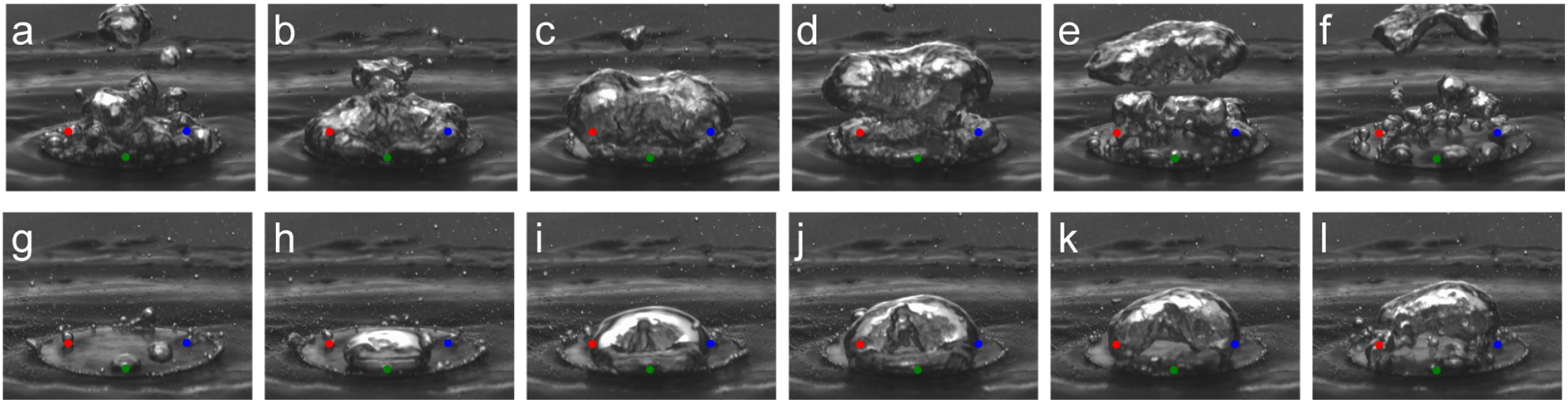

Figure 9. Vapor formation during the onset of acoustic actuation (a-f, elapsed time is 50 msec) and during the asymptotic stage of the actuation ( $\mathrm{g}-1$, elapsed time is $25 \mathrm{msec}$ ) when the centerline of the acoustic beam is incident on the thermocouple sensor TC-3 (green dot) at $\phi=60^{\circ}$. The red, green, and blue dots correspond to the locations of the three thermocouples embedded in the surface.

evaporation apparently occurs regularly at the incidence sensor ostensibly owing to the larger pressure fluctuations along the beam's centerline. Therefore, the longer phase- or time-averaged temperature is lower than at the rest of the surface. Figure $9 \mathrm{~g}$ shows that the left and right thermocouple sensor (blue and red dots) are also acting as preferred nucleation sites for vapor relative to the rest of the surface, albeit not as intensely as at the center (incidence) sensor. Finally, the frames that show the large transitory evaporation occurring in the midst of vapor suppression explain why there are significantly more fluctuations in
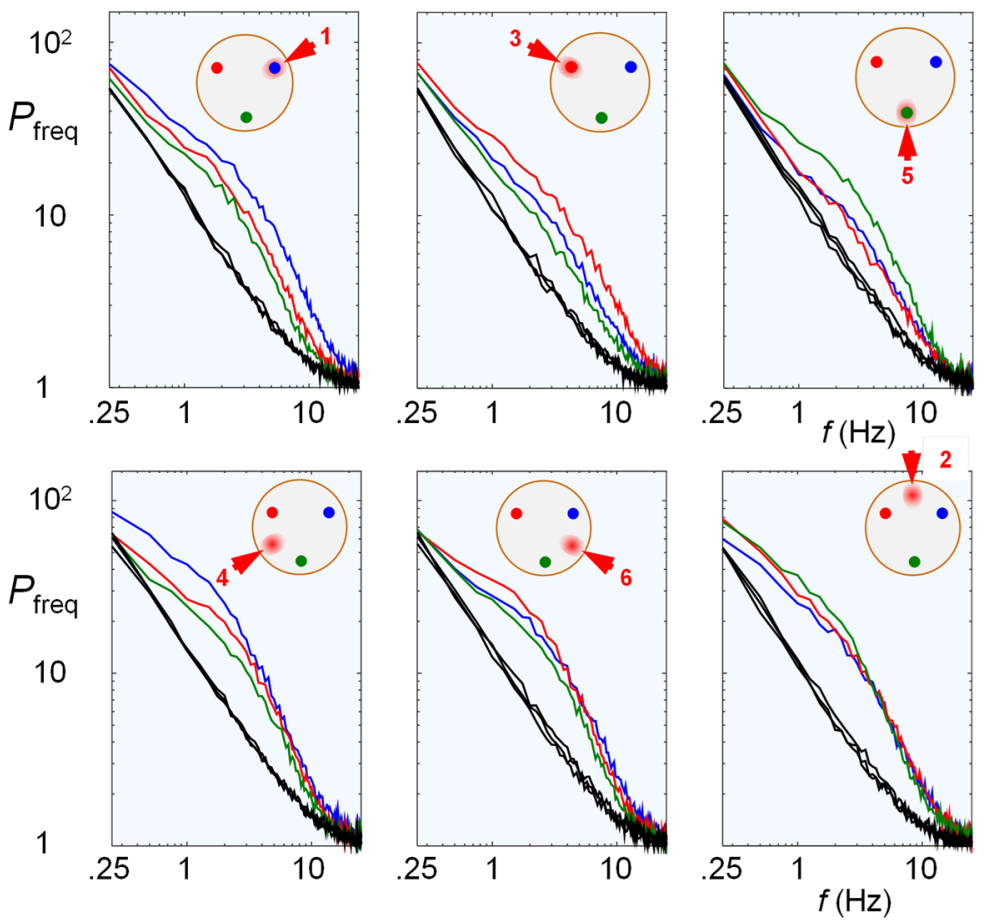

Figure 10. Power spectra of surface superheat $\Delta T_{\mathrm{s}}$ measured by the three surface thermocouple sensors TC-1, 2, and 3 during onset and termination of actuation for six azimuthal positions of the acoustic beam on the heater surface shown schematically by the red circular spot. Spectra in the absence of acoustic actuation for each thermocouple sensor are shown in black, and are essentially equal for all thermocouple sensors at all actuator positions. At each set of the three odd- and even-number azimuthal positions of the acoustic actuator, the acoustic beam is incident either on or between TC-1, 2, and 3. the temperature time traces during actuation compared to the baseline (in the absence of actuation), as evident in the temperature traces in Figure 7.

These temperature fluctuations effects also appear in the frequency domain. Figure 10 shows the power spectra of each surface thermocouple in the presence of actuation (blue, red, green traces - TC-1, 2, 3; respectively) and the absence of actuation (black traces) for all 6 actuator positions. These power spectra curves were generated by averaging over 120 cases for each thermocouple and actuator position. As expected, the fluctuation power for all three thermocouples is the same when actuation is absent, regardless of actuator position (black 
curves). Furthermore, there are no detectable effects beyond $15 \mathrm{~Hz}$, as higher frequencies exceed the transient response capabilities of the thermocouples. As was clear from the time traces in Figure 7, Figure 10 shows that the average power of the fluctuations for all thermocouples is increased in the presence of actuation relative to the baseline, regardless of actuator position relative to the thermocouples. The thermocouple in line with the actuator's incidence location sees the largest fluctuation when the actuator is aimed directly at the thermocouple (Figure 10, top row -curves blue, red, and green respectively from left to right). But interestingly, this also occurs when the actuator is aimed on the far side from the thermocouple (Figure 10, bottom row - blue, red and green respectively from left to right). For the odd-number actuator positions (top row) this is clearly the effect of preferred nucleation at the centered thermocouple sensor as can be seen from images of the high-speed video in Figure 9; the large periodic vapor formation events lead to larger temperature fluctuations at the centered thermocouple sensor compared to its neighbors. For the even-number actuator positions (bottom row), any large, periodic vapor formations lead to temperature fluctuations in the thermocouple sensor on the far-side of beam incidence as the recurring vapor masses are advected across the heater (driven by the ultrasonic actuation) and over the centered thermocouple senor, thereby modulating the local heat transfer.

The tendency of preferred nucleation sites to remain cooler in the presence of acoustic actuation is also prevalent when the actuation is applied to textured boiling surfaces as discussed in Section 6.

\section{THE EFFECTS OF ULTRASONIC ACTUATION ON A MICROMACHINED SURFACE}

The effect of ultrasonic actuation on boiling in the presence of surface microchannels is depicted in Figure 11 (the heat transfer curves for the baseline surface are also included for reference). These data show that while the presence of open microchannels results in a significant reduction in surface superheat, acoustic actuation having a characteristic wavelength that is slightly larger

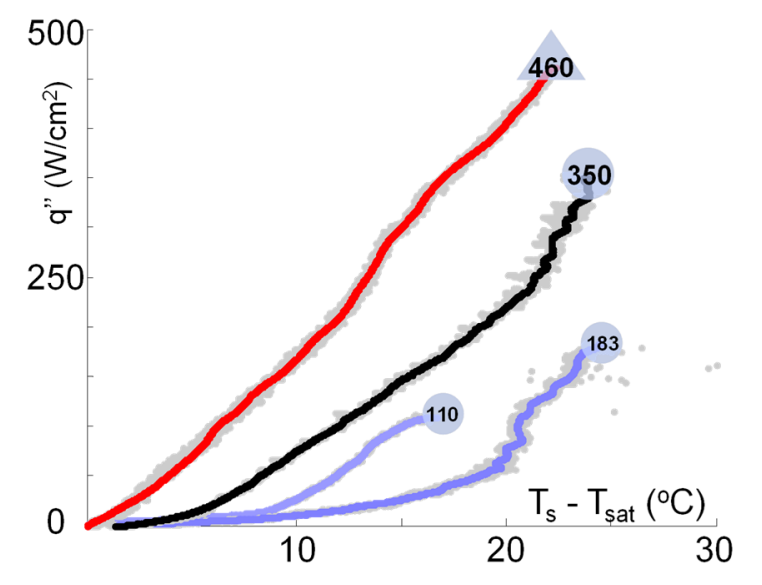

Figure 11. Boiling curves with $400 \mu \mathrm{m}$ channels in the absence and presence of actuation. The corresponding curves for the baseline (plain) surface are also shown for reference (higher CHF is attained with actuation). than the channel width can effectively reduce the surface superheat even further. This reduction implies a significant increase in bubble formation and detachment rates that is accompanied by entrainment of sub-cooled fluid through the channels. It is noteworthy that the reduction in surface superheat in the presence of actuation is in contrast to the effect of actuation on the baseline (plain) surface where actuation results in an increase in surface superheat. It is also noteworthy that ultrasonic actuation leads to a significant increase in the CHF (exceeding 30\%). 


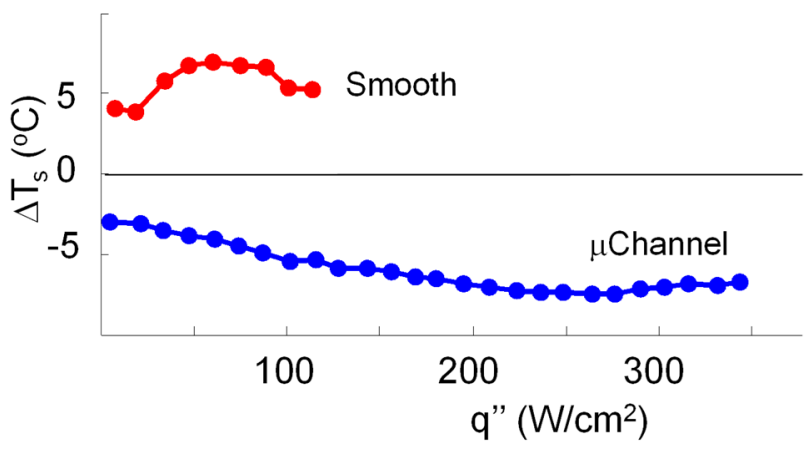

Figure 12. The change in surface superheat versus surface heat flux for the baseline surface and the surface with 400 $\mu \mathrm{m}$ microchannels.
The variation of surface superheat with heat flux in the presence of acoustic actuation is shown in Figure 12 for a surface with $400 \mu \mathrm{m}$ channels and the baseline surface. As noted above, the microchannel grid enables regular formation and detachment of the vapor bubbles along the vertical intersection edges of the microchannels and concurrent entrainment of subcooled liquid along the microchannels towards these intersections. This flow reduces the drag on the rising vapor bubbles and simultaneously increases the heat flux capacity of the surface. The lack of suppression in the rate of evaporation due to the nucleation sites being at the bottom of the microchannels (cf. Section 5), along with the entrainment of subcooled liquid into the microchannels, contribute to the significant reduction in surface superheat for the microchannel surface compared to the increase in surface superheat observed with the plain surface. These effects are the subject of continued investigation.

The boiling process at multiple power levels $\left(100,150,200,250\right.$, and $\left.300 \mathrm{~W} / \mathrm{cm}^{2}\right)$ in the absence and presence of acoustic actuation is compared using high-speed video images (Figure 13). Similar to the effect of the actuation on vapor formation over the plain heater (cf., Figure 5), the actuation breaks up the large vapor concentrations that form above the heater's surface into smaller vapor bubbles. The apparent reduction in vapor mass may be associated with enhanced condensation in the liquid pool as a result of the breakup and the increased vapor-liquid interfaces. It is remarkable that at low power levels the actuation results in clear section of the upper surface of the microchannels (e.g., Figures 13a and f). It should be noted that the lower surface superheat compared to the plain heater can be attributed to a combination of more efficient phase change process, or more effective entrainment of ambient subcooled fluid into the nucleation sites within the channel array. The differences between the vapor
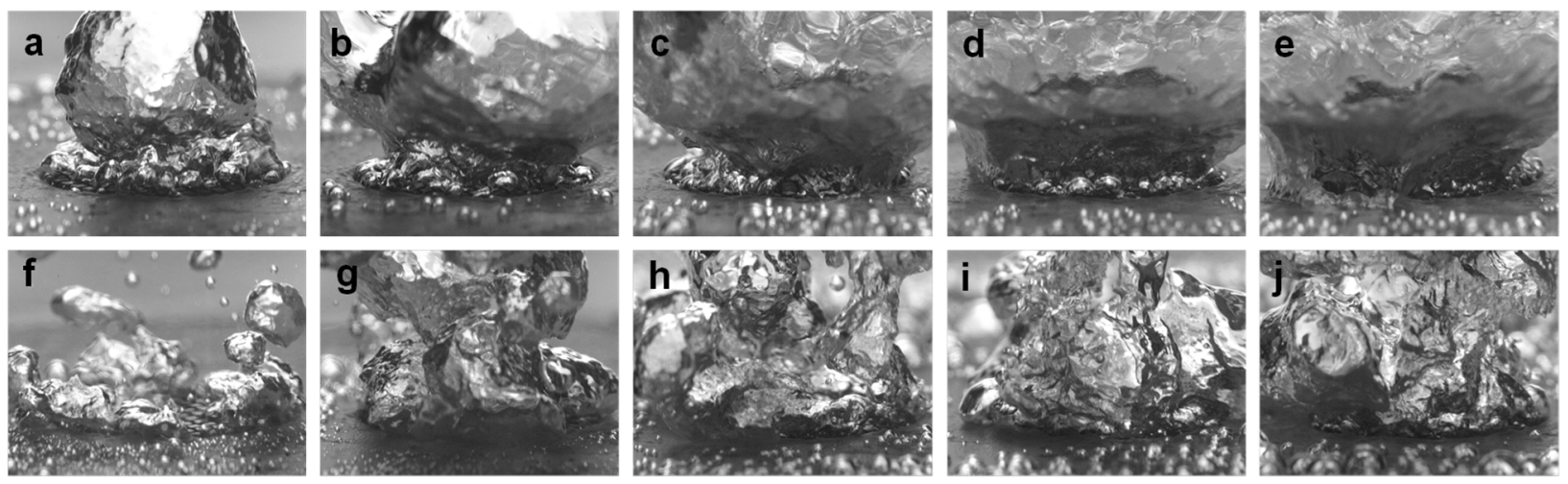

Figure 13. Images from high-speed videos of vapor formation along the heat transfer curves of the heater surface with $400 \mu \mathrm{m}$ wide microchannel (Figure 3) in the absence (top row, a-e) and presence of acoustic actuation (bottom row, f-j) at $100,150,200,250$, and $300 \mathrm{~W} / \mathrm{cm}^{2}$, respectively. 
structure in the absence and presence of actuation are the plain and textured surfaces are apparent even up to $300 \mathrm{~W} / \mathrm{cm}^{2}$ (Figure $13 \mathrm{e}$ and j).

\section{CONCLUSIONS}

Boiling heat transfer from a submerged surface in a subcooled liquid bath is investigated for plain and textured heated surfaces. It is shown that the boiling can be significantly enhanced by arrays of intersecting open microchannels that are machined onto the surface. The microchannel intersections provide distinct, separated vapor nucleation sites and enable the transport of makeup liquid to the boiling sites thereby affecting the evolution and advection of the vapor bubbles. It is shown that the presence of the microchannel grid fundamentally changes the fluid dynamics of the boiling induced flow. The microchannels exploit the motions of the rising vapor bubbles to help pump makeup fluid to the boiling sites. Microchannels also control the location, growth, and detachment of the vapor bubbles, cause a reduction in the mean surface superheat, and lead to a significant increase in the critical heat flux compared to a plain heater surface. Using the heat flux computed based on the projected area $\left(2 \mathrm{~cm}^{2}\right.$ for all surfaces) and the extrapolated surface temperature it is shown that a microchannel array having a pitch of $1.2 \mathrm{~mm}$ and a width to depth ratio of 1:2.5 (400 $\mu \mathrm{m}$ wide) is most effective at reducing the surface superheat and yielding the highest critical heat flux (up to $350 \mathrm{~W} / \mathrm{cm}^{2}$ ) indicating a balance between the flow rate through the microchannels and the preheating of fluid prior to evaporation at the heated surface.

Boiling heat transfer on both the plain and textured surfaces is strongly enhanced by low-power ultrasonic acoustic forcing (having a characteristic wavelength of $900 \mu \mathrm{m}$ ) that controls the formation and evolution of the vapor bubbles and inhibits the instability that leads to film boiling at the critical heat flux. The acoustic actuation induces interfacial forces that affect the bubbles' contact line with the surface leading to their detachment. Furthermore, it induces break-up of the vapor-liquid interface that leads to enhanced condensation of the vapor into the bulk fluid. The effects of the acoustic actuation were first investigated on the planar (baseline) surface and then in the presence of surface microchannels. It is shown that on the plain surface the acoustic actuation increases the surface superheat (up to $7^{\circ} \mathrm{C}$ ) relative to the baseline, and the CHF increases from 110 to 183 $\mathrm{W} / \mathrm{cm}^{2}(65 \%)$. It appears that the increase in surface superheat is caused by the suppression of boiling at most nucleation sites and the removal of small $[\mathrm{O}(10 \mu \mathrm{m})]$ vapor bubbles from preferred, active nucleation sites before they can grow significantly. However, this suppression is diminished at surface protrusions, such as edge discontinuities, or in the presence of surfaceembedded temperature sensors therefore reducing the increase in surface superheat. At the same time, the fast removal of smallscale vapor bubbles from the surface by the actuation-induced body forces contributes to a reduction in the vapor mass and accelerated condensation over the surface and ultimately to an increase in the CHF limit. In the presence of surface microchannels acoustic actuation reduces the surface superheat (compared to an increase in superheat over the plain surface) indicating a significant increase in bubble formation and detachment rates that is accompanied by entrainment of sub-cooled 
fluid through the microchannels. It is clear that the vapor suppression effects seen on a plain surface do not significantly affect the grid of nucleation sites within the microchannels, therefore making the combined use of microchannels and acoustic actuation an attractive approach for practical applications. In the presence of $400 \mu \mathrm{m}$-wide surface microchannels, acoustic actuation leads to a $30 \%$ improvement in the critical heat flux (from $350 \mathrm{~W} / \mathrm{cm}^{2}$ to $460 \mathrm{~W} / \mathrm{cm}^{2}$, in stagnant bulk fluid) while reducing the surface superheat over the entire boiling curve by an average of $7{ }^{\circ} \mathrm{C}$.

\section{FUNDING SOURCE}

A portion of this work was supported by the Office of Naval Research.

\section{REFERENCES}

[1] Zerby M and Kuszewski M 2002 "Final report on next generation thermal management (NGTM) for power electronics," NSWCCD Technical Report TR-82-2002012

[2] Bar-Cohen A 1993 “Thermal management of electronic components with dielectric liquids," JSME B 36 1-25

[3] Mudawar I 2001 "Assessment of high-heat-flux thermal management schemes," IEEE Transactions on Components and Packaging Technology 24 122-41

[4] McGillis W R and Carey V P 1991 "Pool boiling enhancement techniques for water at low pressure," 7th IEEE Semiconductor Thermal Measurement and Management Symposium, 64

[5] Bergles A E 1997 "Enhancement of pool boiling," Int J. Refrig. 20 545-551

[6] Li C Wang Z Wang P-I Peles Y Koratkar N and Peterson G P 2008 "Nanostructured copper interfaces for enhanced boiling," Small 4 1084-1088

[7] Launay S Fedorov A Joshi Y Cao A and Ajayan P 2006 "Hybrid micro-nano structured thermal interfaces for pool boiling heat transfer enhancement," Microelectronics Journal 37 1158-1164

[8] Chien L-H and Webb R L 1998 "Visualization of pool boiling on enhanced surfaces," Experimental Thermal and Fluid Science $16332-341$

[9] Li C and Peterson G P 2007 "Parametric Study of Pool Boiling on Horizontal Highly Conductive Microporous coated surfaces," Journal of Heat Transfer 129 1465-1475

[10] Sloan A Penley S and Wirtz R A 2009 "Sub-Atmospheric Pressure Pool Boiling of Water on a Screen-Laminate Enhanced Surface," 25th IEEE SEMI-THERM Symposium 246-253

[11] Agostini B Fabbri M Park J E Wojtan L Thome J R, and Michel B 2007 "State of the art of high heat flux cooling technologies," Heat Transfer Eng. 28 258-8

[12] Heffington S Tillery S Smith M and Glezer A 2003 "Enhanced boiling heat transfer by submerged, vibration induced jets," Therminic: 9th International, Aix-en-Provence, France 
[13] Tillery S W Heffington S N Smith M K and Glezer A 2006 "Boiling heat transfer enhancement using a submerged, vibration-induced jet," J. Electron. Packag. 128 145-49

[14] Isakoff S E 1956 "Effect of an ultrasonic field on boiling heat transfer - Exploratory investigation," Stanford University Press, Stanford, CA, 15-28

[15] Wong S W and Chon W Y 1967 "Effects of ultrasonic vibrations on burnout heat flux and critical temperature difference," Can. J. Chem. Eng. 45 384-5

[16] Park K A and Bergles A E 1988 "Ultrasonic enhancement of saturated and subcooled pool boiling," Int. J. Heat Mass Trans. $31664-7$

[17] Kim H-Y Kim Y G and Kang B H 2004 "Enhancement of natural convection and pool boiling heat transfer via ultrasonic vibration," Int. J. Heat Mass Trans 47 2831-40

[18] Sitter J S Snyder T J Chung J N and Marston P L 1998 "Acoustic field interaction with a boiling system under terrestial gravity and microgravity," J. Acoustic. Soc. Am. 104 2561-9

[19] Sitter J S Snyder T J Chung J N and Marston P L 1998 "Terrestrial and microgravity pool boiling heat transfer from a wire in an acoust ic field," Int. J. Heat Mass Trans. 41 2143-55

[20] Hao Y Oguz H N and Prosperetti A 2001 "The action of pressure-radiation forces on pulsating vapor bubbles," Phys. of Fluids 13 1167-77

[21] Douglas Z Boziuk T R Smith M K and Glezer A 2012 “Acoustically enhanced boiling heat transfer," Physics of Fluids 24(5)

[22] Li B Han X Wan Z Wang X Tang Y 2016 "Influence of ultrasound on heat transfer of copper tubes with different surface characteristics in sub-cooled boiling," Applied Therm. Eng. 92 93-103

[23] Hetsroni G Moldavsky L Fichman M Pogrebnyak E Mosyak A 2014 "Ultrasonic enhancement of subcooled pool boiling of freely oscillated wires," Int. J. of Multiphase Flow. 67 13-21

[24] Leighton T G 1994 The Acoustic Bubble, Academic, San Diego CA pp. 345-65

[25] Panton R L 2005 Incompressible Flow, John Wiley \& Sons, Inc, Hoboken NJ pp. 646-8

[26] Torr, G. R. "The acoustic radiation force." American Journal of Physics 52, no. 5 (1984): 402-408.

[27] Bunkin F V Kravtsov Y A Lyakhov G A 1986 “Acoustic analogues of nonlinear-optics phenomena," Soviet Physics Uspekhi 29 607-619 